EPJ Web of Conferences 19, 02007 (2012)

DOI: $10.1051 /$ epjconf/20121902007

(C) Owned by the authors, published by EDP Sciences, 2012

\title{
Sub-structures in the inner halo of the Milky Way
}

\author{
A.K. Vivas ${ }^{1, a}$, R. Zinn ${ }^{2}$, S. Duffau ${ }^{3}$ and Y. Jaffé ${ }^{4}$ \\ ${ }^{1}$ Centro de Investigaciones de Astronomía (CIDA), Mérida, Venezuela \\ ${ }^{2}$ Astronomy Department, Yale University, New Haven, CT, USA \\ ${ }^{3}$ Astronomisches Rechen-Institut, Zentrum für Astronomie der Universität Heidelberg, \\ Germany \\ ${ }^{4}$ Osservatorio Astronomico di Padova INAF, Italy
}

\begin{abstract}
We present a spectroscopic study of a sample of 238 RR Lyrae stars, from the QUEST survey, located in the Galactic halo at distances between 4 and $20 \mathrm{kpc}$ from the Sun. Combining their spatial position and kinematics we were able to identify sub-structures in this part of the halo. Some of those sub-structures may be associated with known halo features like the Virgo Overdensity, the Hercules-Aquila Cloud, and the Anticenter Stream.
\end{abstract}

\section{INTRODUCTION}

RR Lyrae stars are excellent standard candles; their distances can be obtained with a precision of $7 \%$. The spatial distribution alone may reveal sub-structures in the halo. However, smaller groups may only be detected when combining the spatial distribution with kinematics. In order to study the phase space distribution of the stars in the halo, we have obtained spectroscopy of a large number of QUEST RR Lyrae stars [1] using different telescopes in Chile and the US. We measured radial velocities with precision of $16 \mathrm{~km} / \mathrm{s}$, using the methods described in [2]. We have obtained spectra of $85 \%$ of all QUEST RR Lyrae stars brighter than $\mathrm{Vo}=16.0$. Fainter stars have been observed in specific regions of the sky like the Virgo overdensity. Our spectroscopic sample contains 238 RR Lyrae stars.

\section{FINDING SUB-STRUCTURES}

We searched for stellar pairs among the RR Lyrae stars. Two stars make a pair when they are close in the sky and have a similar radial velocity. A group is formed when two or more pairs have stars in common. For the formal definition of a pair we used two methods which gave very similar results: (i) the 4 distance parameter as defined by [3], and (ii) we imposed a maximum separation in distance and velocity to define a pair. In order to study if the resulting groups may be just random fluctuations of halo stars, we created simulated random samples using the number density radial profile of RR Lyrae stars in the halo [4] and expected velocity distribution [5]. We then applied the group finding algorithms to the simulated samples and counted how many times we detected a group with similar properties as the ones found in the spectroscopic sample. In Figure 1 we show, with color symbols, those stars belonging to significant groups in the halo. The large circles indicate groups with a high probability of being real since

\footnotetext{
ae-mail: akvivas@cida.ve
}

This is an Open Access article distributed under the terms of the Creative Commons Attribution-Noncommercial License 3.0, which permits unrestricted use, distribution, and reproduction in any noncommercial medium, provided the original work is properly cited. 


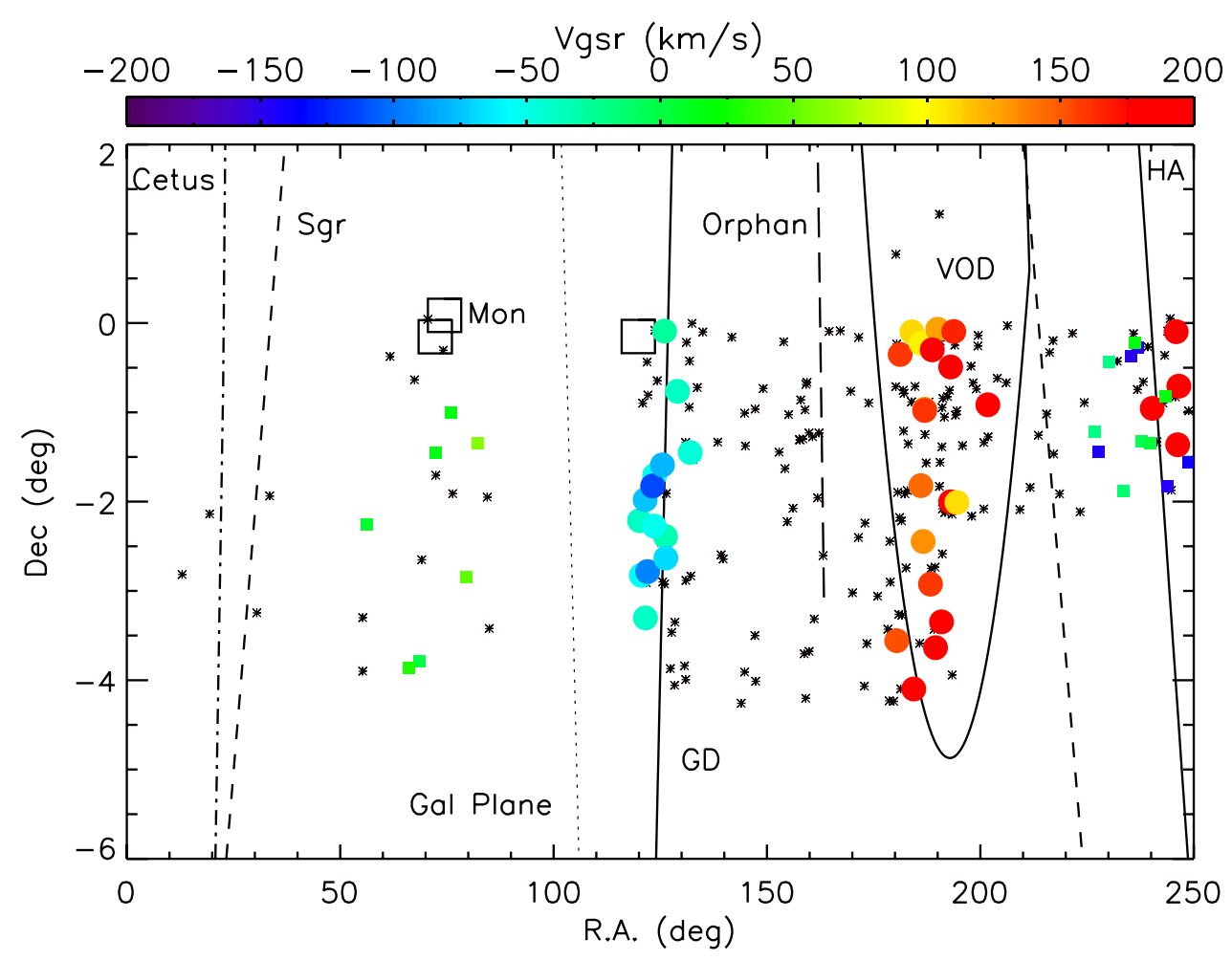

Figure 1. Distribution in the sky of the QUEST RR Lyrae stars with spectroscopy. The color symbols show RR Lyrae stars belonging to groups. For reference we have shown with different lines the Sagittarius Plane (Sgr), the Orphan, Cetus and Grillmair-Dionatos (GD) streams, the Hercules-Aquila cloud (HA), the Virgo Overdensity region (VOD), and detections of the Monoceros ring (Mon) in the region. With the method described above we have detected two significant groups in the VOD region with velocities (in the galactic standard of rest) of 142 and $221 \mathrm{~km} / \mathrm{s}$. The first one is located at a mean distance of $19 \mathrm{kpc}$, and corresponds to the Virgo Stellar Stream [6]. The second group has stars between 8 and $16 \mathrm{kpc}$. A third group with lower probability was also found at $-86 \mathrm{~km} / \mathrm{s}$, but it is not shown here for clarity. In the region of the Hecules-Aquila cloud we detected three different kinematical groups with $V_{g s r}=185,-146$ and $-3 \mathrm{~km} / \mathrm{s}$, in the distance range from 4 to $11 \mathrm{kpc}$ from the Sun. We have found also a significant group along the GD stream, but its velocity $(-59 \mathrm{~km} / \mathrm{s})$ does not agree with the detection by [7] in about the same distance range and region of the sky. Finally a medium probability group was found at 5-10 kpc near the Monoceros ring detections, at RA $\sim 80^{\circ}$. However, the Monoceros ring should be more distant than our spectroscopic limit in that part of the sky.

$<2 \%$ of the 10.000 simulations produced similar groups. The small squares have medium probability (similar groups were produced in 2 to $5 \%$ of the simulations). The smaller, black symbols are stars not belonging to any group. For the groups, the color code indicates the velocity of the RR Lyrae stars. For reference, we have indicated the location of the most prominent known halo sub-structures in this part of the sky.

\section{References}

[1] A.K. Vivas, R. Zinn, C. Abad, P. Andrews, C. Bailyn, C. Baltay, A. Bongiovanni, C. Briceño, G. Bruzual, P. Coppi et al., AJ, 127, 1158 (2004)

[2] A.K. Vivas, Y.L. Jaffé, R. Zinn, R. Winnick, S. Duffau, C. Mateu, AJ, 136, 1645 (2008) 
Assembling the Puzzle of the Milky Way

[3] E. Starkenburg, A. Helmi, H.L. Morrison, P. Harding, H. van Woerden, M. Mateo, E.W. Olszewski, T. Sivarani, J.E. Norris, K.C. Freeman et al., ApJ, 698, 567 (2009)

[4] A.K. Vivas, R. Zinn, AJ, 132, 714 (2006)

[5] W.R. Brown, M.J. Geller, S.J. Kenyon, A. Diaferio, AJ, 139, 59 (2010)

[6] S. Duffau, R. Zinn, A.K. Vivas, G. Carraro, R.A. Méndez, R. Winnick, C. Gallart, ApJL, 636, L97 (2006)

[7] B.A. Willett, H.J. Newberg, H. Zhang, B. Yanny, T.C. Beers, ApJ, 697, 207 (2009) 